\title{
Automatic Recognition by Gait
}

\author{
Recognizing people by the way they walk promises to be useful for identifying \\ individuals from a distance; improved techniques are under development.
}

\author{
By Mark S. Nixon, Member IEEE, and John N. Carter, Member IEEE
}

\begin{abstract}
Recognizing people by gait has a unique advantage over other biometrics: it has potential for use at a distance when other biometrics might be at too low a resolution, or might be obscured. The current state of the art can achieve over $90 \%$ identification rate under situations where the training and test data are captured under similar conditions, while recognition rates with change of clothing, shoe, surface, illumination, and pose usually decrease performance and are the subject of much of the current study. Recognition can be achieved on outdoor data with uncontrolled illumination and at a distance when other biometrics could not be used. We shall show how this position has been achieved, covering most approaches to recognition by gait and the databases on which performance has been evaluated. We shall describe the context of these approaches, show how recognition by gait can be achieved and how current limits on performance are understood. We shall describe results on the most popular database, showing how recognition can handle some of the covariates that can affect recognition. We shall also investigate the supporting literature for this research, since the notion that people can be recognized by gait has support not only in medicine and biomedicine, and also in literature and psychology and other areas. In this way, we shall show that this new biometric has capability and research and application potential in other domains.
\end{abstract}

KEYWORDS | Biometrics; covariate factors; gait; gait analysis; gait database; gait recognition

\section{INTRODUCTION}

A unique advantage of gait as a biometric is that it offers potential for recognition at a distance or at low resolution when the human subject occupies too few image pixels for other biometrics to be perceivable. In gait recognition, we seek to process video images, to derive numbers that reflect the identity of the moving subject. By using a

Manuscript received June 6, 2006; revised August 21, 2006

The authors are with the School of Electronics and Computer Science, University of Southampton, SO17 1BJ Southampton, U.K. (e-mail: msn@ecs.soton.ac.uk; jnc@ecs.soton.ac.uk).

Digital Object Identifier: 10.1109/JPROC.2006.886018 silhouette, a subject is described not just by shape but also by motion; an alternative is to model features, here limb movement. A selection of approaches to automatic recognition by gait is shown in Fig. 1. The taxonomy is not as clear as the diagram suggests since a number of approaches straddle the boundaries. Moving shape techniques are mainly silhouette-based approaches and later approaches used the motion (the sequence of the silhouette's appearance).

The current state of the art is that databases of over 100 subjects imaged walking outdoors can be recognized with well over $90 \%$ identification rate (equaling many biometrics) and factors which affect gait are understood, there is capability to handle application environment and understanding of the measures' potency for recognition purposes. An example of the progression in performance is shown in Table 1 , for classification by the $k$-nearest neighbor on datasets of increasing size. Here, the (Southampton) databases existing around 2001 were near 30 subjects, rising to over 100 subjects later. This performance level has been maintained since the earliest approaches.

Recognition by gait is actually one of the newest biometrics, since its development only started when computer memory and processing speed became sufficient to process sequences of image data with reasonable performance. Recognition approaches to gait were first developed in the early 1990s and were evaluated on smaller databases than those in Table 1, and showed promise-these are the techniques prior to the horizontal divide in Fig. 1. DARPA's Human ID at a Distance program [31] then collected a rich variety of data and developed a wide variety of technique and showed not only that gait could be extended to large databases and could handle covariate factors. Since the DARPA program, research has continued to extend and develop technique, with especial consideration of practical factors such as feature potency.

Naturally, its development has benefit from complementary studies which support the notion of gait as a biometric: there is considerable evidence in biomechanics, psychology, and literature for the notion that people can be recognized by the way they walk. Naturally, this survey is as inclusive as possible, covering most main approaches to 


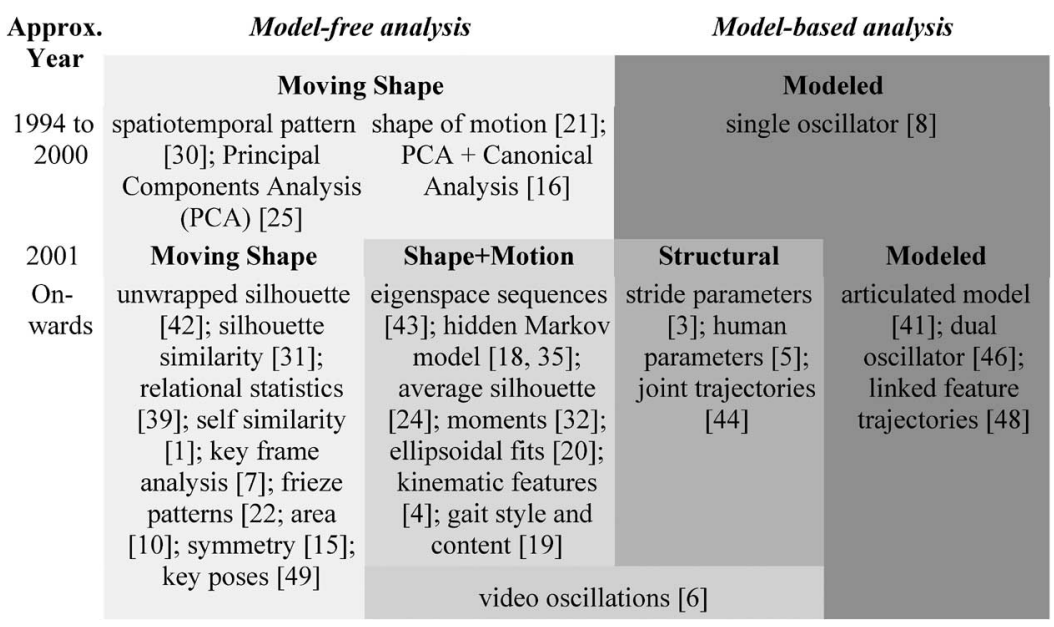

Fig. 1. Approaches to automatic gait recognition.

automatic recognition by gait, its performance, and background studies; much greater detail and context is available elsewhere [29].

\section{SUBJECTS ALLIED TO GAIT}

\section{A. Literature}

The usual meaning of "gait" is "manner of walking," though this is sometimes given as a "manner of moving on foot," since this can subsume running. It is mainly given as derived from the Middle English gate, meaning path or gait, as derived from the Old Norse gata, meaning path. Shakespeare made several references to the individuality of gait, e.g., in Henry IV Part II "To seem like him: so that, in speech, in gait, in diet, in affections of delight, in military rules, humours of blood, he was the mark and glass, copy and book."

\section{B. Other Gait Analysis}

1) Medical and Biomechanical Analysis: The biomechanics literature makes observations concerning identity: "A given person will perform his or her walking pattern [Fig. 2] in a fairly repeatable and characteristic way, sufficiently unique that it is possible to recognize a person at a distance by their gait" [45]. The aim of medical research has been to classify the components of gait for the treatment of pathologically abnormal patients. Murray et al. [28] produced standard movement patterns for pathologically normal people which were used to compare the gait patterns for pathologically abnormal patients [27]. In all there appear to be 20 distinct gait components, some of which can only be measured from an overhead view of the subject. Murray found "the pelvic and thorax rotations to be highly variable from one subject to another" [27]. These patterns would be difficult to measure even from an overhead view of the subject, which would not be suited to application in many practical situations and, unlike biometrics, these studies required markers attached to the subject.

The rotation of the inclination of the thigh, Fig. 3(a), is characterized by one period of extension and one period of flexion in every gait cycle. Fig. 3(b) gives the average rotation pattern [27] where the upper and lower lines indicate standard deviation. In the first half of the gait cycle, the hip is in continuous extension as the trunk moves forward over the supporting limb. In the second half, once the weight has been passed onto the other limb, the hip flexes in preparation for the swing phase. This flexing action accelerates the hip directing the swinging limb forward for the next step.

Running has significant biomechanical differences from walking and you cannot just walk fast to claim that

Table 1 Progression of Gait Recognition Results by Symmetry [14]

\begin{tabular}{|c|c|c|c|}
\hline \multirow{2}{*}{ Number of Subjects } & No. of Sequences & \multicolumn{2}{|c|}{ Correct Classification Result (\%) } \\
\cline { 3 - 4 } & (each with 1 gait cycle) & $k=1$ & $k=3$ \\
\hline 28 & 4 & 97 & 96 \\
\hline 50 & 4 & 95 & 93 \\
\hline 114 & 8 & 94 & 90 \\
\hline
\end{tabular}




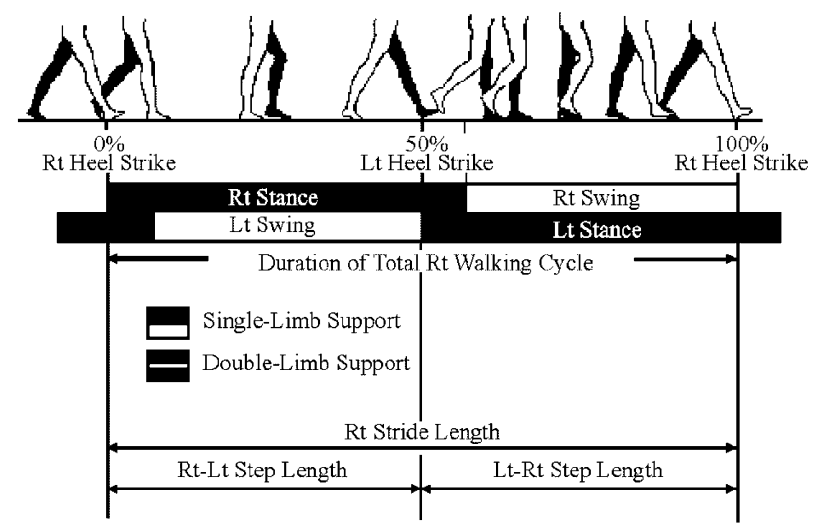

Fig. 2. The walking cycle.

you are running. By biomechanics definitions, walking and running are distinguished firstly by the stride duration, stride length, velocities, and the range of motion made by the limbs. That is, the kinematics of running differ from those of walking where the joints' motion increases significantly as the velocity increases. A second difference concerns the existence of periods when neither foot is in contact with the ground, which does not occur in walking.

2) Variation in Gait/Covariate Factors: Load and footwear can affect gait as can alcohol. Naturally, tight clothing can affect gait, whereas loose-fit clothing can affect the perception of gait by video. Intuitively, gait will change with age as do most biometrics, except ears. These changes can be due to compound changes in physiology, neurology, and/or illness. Without rapid and convenient analysis, it is unlikely that study of effect of aging will progress much further and this is one area where automated gait analysis via computer vision can make contributions beyond those associated with biometric issues.

\section{Psychology}

Psychological studies support the notion that gait can be perceived by human vision as unique. In early studies [17] participants were presented with images produced from points of light attached to body joints, as in Fig. 4 . When the points were viewed in static images (one of the frames in Fig. 4) they were not perceived to be in human form, rather that they formed a picture - of a Christmas tree even. Motion is the recognition cue, without it all that is perceived is a random pattern of points. When the points (the frames) were animated, they were immediately perceived as representing a human in motion. Later work showed how by point light displays a human could be rapidly extracted and that different types of motion could be discriminated, including jumping and dancing.

One early study showed how gender could be perceived, attributed to anatomical differences which result in greater shoulder swing for men, and more hip swing for women. Indeed, a torso index (the hip shoulder ratio) has been shown to discriminate gender and the identification of gender by motion of the center of movement was also suggested. Gender identification would appear to be less demanding than person identification which progressed to showing how we could identify friends [9]. Essentially, research into the psychology of gait has not received much attention, especially using video, in contrast with the enormous attention paid to face recognition. One more recent study showed [34], using video rather than point light displays, that humans can indeed recognize people by their gait, and to learn their gait for recognition, but again the databases are much smaller than the $100+$ subjects in modern gait-biometric databases.

\section{GAIT DATABASES}

In any pattern recognition study, the database used for evaluation will reveal potency of recognition. Primary concerns include uniqueness and practicality, which are usually revealed by the number of subjects in the database and the imaging conditions, respectively. If the primary purpose of the database is to evidence basic practicality, then data can be derived in a laboratory scenario; if the main aim is to show that it can be achieved by computer vision, then the data can be derived by filming subjects outdoors. Databases seek to include enough samples of enough subjects to allow for an estimate of inter- and intrasubject variation. The data described here was developed especially for purposes of evaluation and is usually freely available for evaluation.

The current databases have a number of subjects which is smaller than that currently used in performance comparison in other biometrics (like face and fingerprint). The databases do include covariate factors and application potential. A more detailed description of some of the databases currently available, and their construction, focuses on their use in an analysis of performance [31]. They

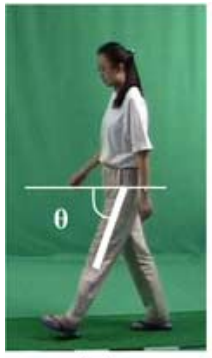

(a)

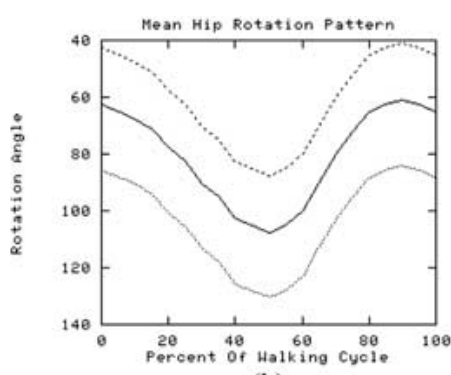

(b)
Fig. 3. Hip inclination: measurement and pattern. (a) Measured hip inclination. (b) Variation with time. 

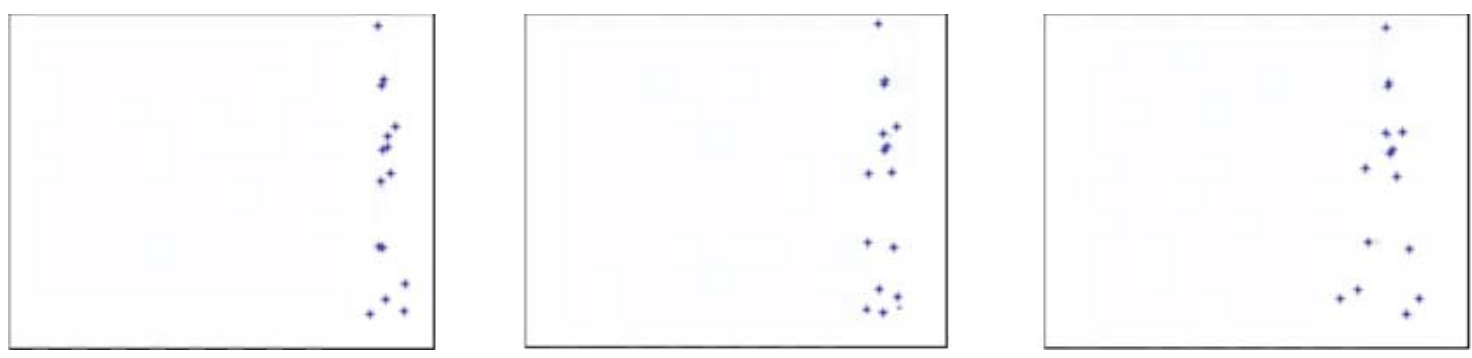

Fig. 4. Marker positions in gait analysis.

include the Universities of Maryland (UMD) — which is outdoor data, simulating a surveillance scenario, National Institute of Standards and Technology (NIST)/South Florida (USF), described next, Southampton [33] which combined outdoor with indoor (video) ground truth by chromakey, and Carnegie Mellon University's (CMU's) Mobo data which is multiview of subjects walking indoors on a treadmill [12].

The NIST/USF Gait Challenge database consisted [31] of 1870 sequences from 122 individuals, with video collected for each individual for two camera views in differing surface conditions and shoe types. The data was collected outdoors, reflecting the added complications of shadows from sunlight, motion in the background, and moving shadows due to cloud cover. This database was the largest then available in terms of number of people, number of video sequences, and variety of conditions under which a person's gait was collected. This database has been used much for evaluation and comparison of performance. One restriction is that there is little repetition within the data, restricting analysis of within-subject (intraclass) variation.

Each subject walked around two similar sized elliptical courses, one on concrete and the other on a grass lawn, Fig. 5(c). Each course was viewed by two cameras located about $15 \mathrm{~m}$ from each end of the ellipse. Information recorded in addition to the video includes sex (75\% male), age (19-54 years), height (1.47-1.91 m), weight (43.1$122.6 \mathrm{~kg}$ ), foot dominance (mostly right), type of shoes (sneakers, sandal, etc.), and heel height. A little over half of the subjects walked in two different shoe types. Thus, for each subject there were up to eight video sequences: (grass $(G)$ or concrete $(C)) \times($ two cameras, $L$ or $R) \times$ (shoe $A$ or shoe $B$ ). Two separate data collection sessions were held, in May and November. The dataset is quite demanding for other biometrics, since in some cases the only biometric that can be seen is gait, as in Fig. 5(a), and the imagery is wholly outdoors and the lighting is uncontrolled. Clearly, face recognition, indeed any biometric analysis, on this data would be a taxing exercise. Originally, the gait challenge concerned analysis of the data for which no briefcase was carried and later data was added for subjects carrying a briefcase.

A later study used manual labeling [23] to gain insight into the relationship between recognition capability and silhouette quality. Silhouettes were created for one gait cycle for 71 subjects under four different conditions, (shoe type, surface, and time) and each pixel was also labeled according to body segment. An example is shown in Fig. 5(b). This allows for understanding of the contribution to recognition capability not only of body labeling, but also of the segments.

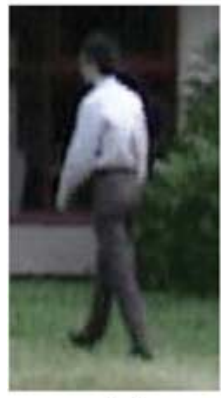

(a)

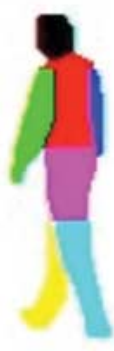

(b)

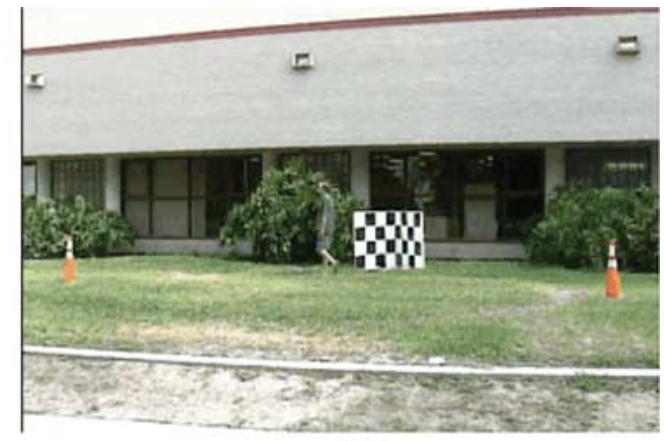

(c)

Fig. 5. NIST/USF gait challenge data. (a) Example subject. (b) Manually labeled. (c) General view. 


\section{SILHOUETTE-BASED APPROACHES}

\section{A. Current Approaches}

The approaches derive the human silhouette by separating the moving object from the background. Then, the subject can be recognized by measurements that reflect shape and/or movement. Some techniques impose a model of the gait sequence, and process a period of gait information whereas others derive the measures for a long sequence of images. As we shall find, the simplest approach is to simply form an average of the silhouette whereas the more complex impose a model on the motion. We shall illustrate this range by considering techniques that have operated on extensive data, or multiple datasets.

A prime example of a model-free approach is two approaches from UMD: Kale et al. and Sundaresan et al.'s deployment of hidden Markov models (HMM) [18], [35] which consider two different image features: the width of the outer contour of a binarized silhouette and the entire binary silhouette itself. An indirect approach to forming a feature vector uses a frame to exemplar distance (FED) which captures a subject's shape and their motion, under the assumption that the camera is sufficiently distant that the moving subject can be considered to be planar. The information in the FED vector sequences is captured using an HMM. A direct approach used the feature vector directly (as opposed to computing the FED) for training an HMM. The observation probability is estimated using the distance between the exemplars and the image features. Results analyzed on the Mobo, UMD, and USF/NIST databases reveal good performance for gait recognition. Rather than repeat similar performance figures for each technique, the performance is usually of the capability that recognition rates exceeding $90 \%$ are achieved. As in any deployment of pattern recognition one can raise capability to $100 \%$ on a selected dataset by specialized tuning.

BenAbdelkader et al.'s approach using self similarity and structural stride parameters (stride and cadence) [1] used PCA applied to self-similarity plots that are derived by differencing. The self-similarity matrix encodes the frequency and phase of gait and thus preserves the dynamics of gait. Classification was performed by $k$-nearest neighbor and evaluated on the UCSD data showing that recognition could be achieved. An extended analysis confirmed performance on the Mobo and UMD data [2].

Sarkar et al.'s approach performs recognition by temporal correlation of silhouettes [31]. The aim was to develop a technique against which future performance could be evaluated (Section IV-B). This was achieved by semiautomatic definition of a bounding box from which a silhouette was matched. Then, the gait period was estimated for use in partitioning sequences for temporal classification. The approach was then evaluated on the Mobo data and on the NIST/USF data, in comparison with a selection of other approaches. This allowed determination of the covariates with the greatest impact, which in one test turned out to be the surface on which subjects walked and the time interval between data recording. The influence of the surface might be that the probe was selected as grass which is rather more unforgiving (uneven) than the concrete surface.

Vega et al. use the change in the relational statistics among the detected image features (which can handle running too) [39] and which removes the need for object models, perfect segmentation, or part-level tracking. The relational statistics are modeled using the probability that a random group of features in an image would exhibit a particular relation. These distributions are represented in a space of probability functions, where the Euclidean distance is related to the Bhattacharya distance between probability functions. Different motion types sweep out different traces in this space. As with other approaches, this is generic to motion analysis and is used to recognize by gait with evaluation on an early version of the NIST/ USF data with high confidence, especially with respect to change in viewpoint. Liu et al. have also developed an average silhouette [24] which is perhaps the simplest recognition feature and had also been used by Veres et al. [40] and by Han et al. [13], though in a study of potency (Section IV-C). Recognition used the Euclidean distance between the averaged silhouette representations and the technique was shown to be considerably faster than the baseline algorithm. Experiments with portions of the average silhouette representation showed that recognition power is not entirely derived from upper body shape; rather the dynamics of the legs also contribute equally to recognition. As will be considered later on analysis of potency, it then raises a feature selection problem: by what can one, or should one, recognize gait?

Collins et al. used key frame analysis for sequence matching [7] with innate viewpoint dependence. The key frames were compared to training frames using normalized correlation, and subject classification was performed by nearest neighbor matching among correlation scores. The approach implicitly captures biometric shape cues such as body height, width, and body-part proportions, as well as gait cues such as stride length and amount of arm swing. The approach was evaluated on the Mobo dataset, and on early versions of the UMD and Southampton and MIT databases showing excellent performance. In another approach from CMU, Liu et al. used "frieze patterns" [22] derived from image sequences by compressing images into a concatenated pattern, with some similarity to the earliest approach [30]. Later, Tolliver et al. were to show [37] that people could be recognized by shape with especial consideration of noisy silhouettes.

The University of Southampton's newer approaches range from a baseline-type approach by Foster et al.'s technique measuring area [10], to extension of technique for object description including symmetry by HayfronAcquah et al. [15] (with some justification from psychology studies) and Shutler et al.'s statistical moments [32]. These 
integrate shape with motion, explicitly considering measures derived from a single period in the gait cycle. Lee et al. used ellipsoidal fits to human silhouettes [20]. Evaluation was performed on the MIT and the Mobo data, also with consideration of gender classification (which was achieved) and of potency for gender classification (for which the thigh orientation was ranked as most potent).

Wang et al. developed an eigenspace transformation of an unwrapped human silhouette [42]. For each image sequence, an improved background subtraction procedure is used to extract moving silhouettes of the walking figure from the background. Temporal changes of the detected silhouettes were then represented as an associated sequence of complex vector configurations in a common coordinate frame, and were further analyzed using Procrustes analysis to obtain the mean shape for use as a gait signature. The full Procrustes distance measure was used for classification by nearest neighbor techniques. This implicitly uses the action of walking to capture the structural characteristics of gait, especially the shape cues. The technique was naturally dependent on clothing and on view, a factor common to most silhouette based techniques. Almost contemporaneously, CASIA used eigenspace transformation of distance signals derived from sequences of silhouettes [43].

Bhanu et al. used kinematic and stationary features [4] by estimating three-dimensional (3-D) walking parameters by fitting a 3-D kinematic model to two-dimensional (2-D) silhouettes. Shape and structure was extracted separately and then combined for recognition. Han et al. [13] were also to use the Gait Energy Image formed by averaging silhouettes and then deployed PCA and multiple discriminant analysis to learn features for fusion. These were deployed to good effect on the NIST data, with performance generally exceeding the baseline algorithm.

Of the more recent approaches, Zhao et al. [49] used the mean amplitude of key poses, evaluated on the NIST/ USF gait challenge data, to achieve recognition. Lee et al. separates gait into style and content by generating temporally aligned gait sequences via local linear embedding with separation by a bilinear model and achieved good performance on the gait challenge database [19]. Boyd's more recent approach straddles model-based and modelfree approaches, by synchronizing the oscillation of pixel intensity with those of arrays of phase-locked loops [6] with patterns analyzed by Procrustes Analysis and directional statistics, and evaluated on Carnegie-Mellon Mobo and the Southampton databases.

\section{B. Results on the NIST/USF Gait Challenge Data}

The NIST data contains different covariate factors: view concerns change in viewpoint, shoe concerns change in footwear, surface concerns the nature of the surface the subject walked on (either grass or concrete), time concerns imagery of the same subject gathered at a different time. Results here are for an automated technique, based on averaging over a single gait cycle [11] with automatic background/foreground segmentation, subject tracking, period detection, and silhouette generation. The results here are for identification PI quoted for the closest match (rank 1) and the closest match within the five nearest matches (rank 5), using a subset of the gait challenge data, compared with those by the baseline approach [31]. Recognition was best for change in viewpoint and for which at best $75 \%$ identification rate and $98 \%$ correct for the subject to be within the five closest matches. Certainly, tuning the analysis can improve the results; the issue here is more the variation over the covariate factors. Clearly, recognition can be achieved over most of the covariates represented in the gait challenge data and this pattern of performance is repeated over many studies using the NIST data [31]. This analysis is demanding in that for outdoor images the feature vector describes an average silhouette automatically derived for a single gait cycle which is then matched to the feature vector describing a single cycle automatically derived from a different sequence. In contrast the baseline approach used template matching, with manual intervention. This provided a minimum performance which a selection of automated approaches rivaled on an earlier database release [31].

The receiver operator characteristic is shown in Fig. 6, which reflects the analysis in Table 2 for the same covariates. These are labeled from a to $l$ and it can be seen that recognition over different views quickly rises to $100 \%$ (trace a) whereas recognition (at the lowest extreme) over time only just reaches $100 \%$ over all samples (trace 1 ). There are many facets which complicate comparison of performance, ranging from the data itself, to implementation of technique - especially on automated template generation, that exacerbate problems in comparing recognition performance, but the Gait Challenge analysis certainly showed that recognition by gait could be achieved on outdoor data (on data where other biometrics could not be deployed) and that some covariates could affect recognition more adversely than others.

\section{Data Potency}

First the Southampton databases (which include covariates) were analyzed separately using ANOVA and PCA to find out which image information (features) is redundant, which features have a relatively high variation between the subjects, and how the original feature set could be reduced without reduction in the varianceexplained and the recognition rate. All databases have redundant features and they are not necessarily all the same. This is important in applications, since it suggests areas on which a camera or feature extraction approach might concentrate. However, to jointly compare disparate databases, the datasets have to be reduced to the same number of features. Therefore, the shared important features between three databases were determined and we 

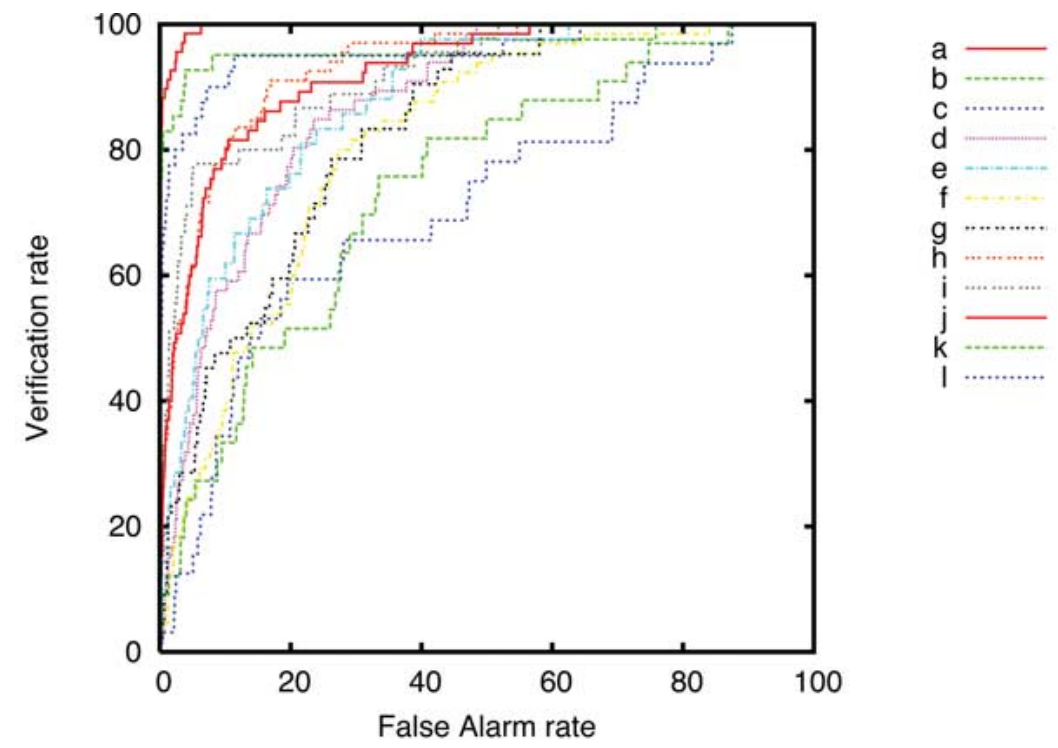

Fig. 6. Receiver operator characteristic: averaged silhouette on gait challenge data.

investigated how reduction in these features could affect recognition. The recognition rate was calculated using Euclidean distance and the nearest neighbor rule. A more sophisticated classifier was not used, since the important factor was only the relative reduction/increase in recognition rate at this stage.

Two sets of important features, which are the same in all three databases, were considered. First, the features which explain $100 \%$ of variance in each data set, i.e., 236, 1001, and 217 features from the three databases. These features contain 115 shared among the datasets. Fig. 7 shows the location of shared 115 features on silhouette at the left top picture. The shared features cover the contours of head, body, some legs, and some features of arm. The bottom left picture in Fig. 7 shows how recognition rate changes with adding additional features. Again here the solid line describes dependency of significant features versus recognition rate (46.3\% for 115 features), while the dashed line corresponds to recognition rate when all features are considered (56.4\%). In this case $17.9 \%$ of recognition rate was lost. Then, further reduction was tried. From each dataset 150 features obtained by PCA earlier were compared and 79 shared features were selected. It was found out that 79 features explain approximately $84 \%$ of variance in each database. These features were projected back onto silhouette and presented in Fig. 7, top right. In this case the most important shared features are contours of the head and body. The recognition rate versus the shared features is presented in bottom right picture of Fig. 7. In this case recognition rate for 79 features was $41.3 \%$ in comparison to $56.4 \%$ for all 4096 features, i.e., a reduction of $26.8 \%$. Practically it

Table 2 Gait Challenge Analysis: Baseline Versus Averaged Silhouette

\begin{tabular}{|c|c|c|c|c|cc|}
\hline \multirow{2}{*}{ Exp. } & Gallery & Covariate & \multicolumn{2}{|c|}{ Baseline $P I(\%)$} & \multicolumn{2}{c|}{ Average silhouette $P I(\%)$} \\
\cline { 4 - 7 } & Size & & Rank 1 & 5 & 1 & 5 \\
\hline a & 68 & View & 73 & 88 & 75 & 98 \\
b & 41 & Shoe & 78 & 93 & 51 & 74 \\
c & 40 & Shoe, View & 48 & 78 & 46 & 78 \\
d & 70 & Surface & 32 & 66 & 19 & 57 \\
e & 44 & Surface, Shoe & 22 & 55 & 8 & 36 \\
f & 69 & Surface, View & 17 & 42 & 3 & 35 \\
g & 44 & Surface, Shoe, View & 17 & 38 & 10 & 46 \\
h & 70 & Briefcase & 61 & 85 & 30 & 67 \\
i & 47 & Briefcase, Shoe & 57 & 78 & 45 & 76 \\
j & 68 & Briefcase, View & 36 & 62 & 25 & 72 \\
k & 33 & Time, Shoe, Clothes & 3 & 12 & 3 & 20 \\
l & 33 & Surface, Time, Shoe, & 3 & 15 & 0 & 17 \\
& & Clothes & & & & \\
\hline
\end{tabular}



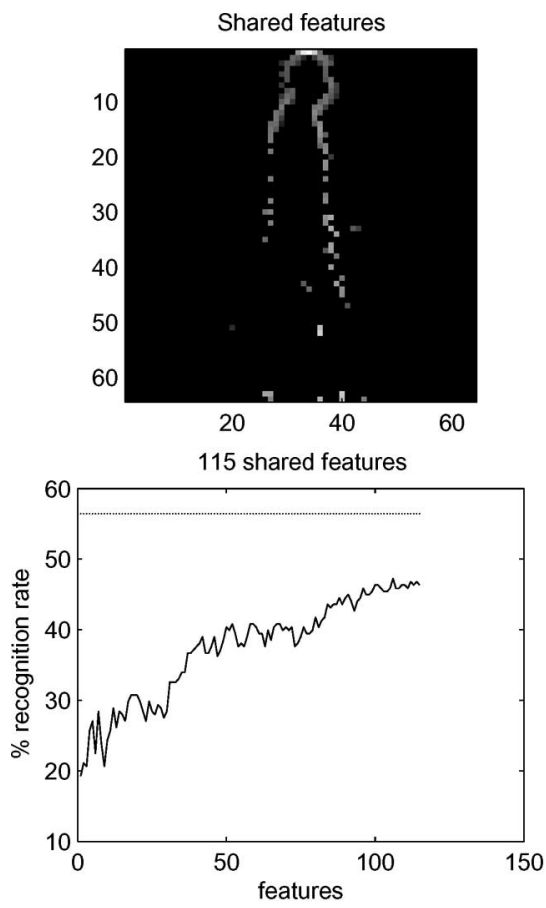
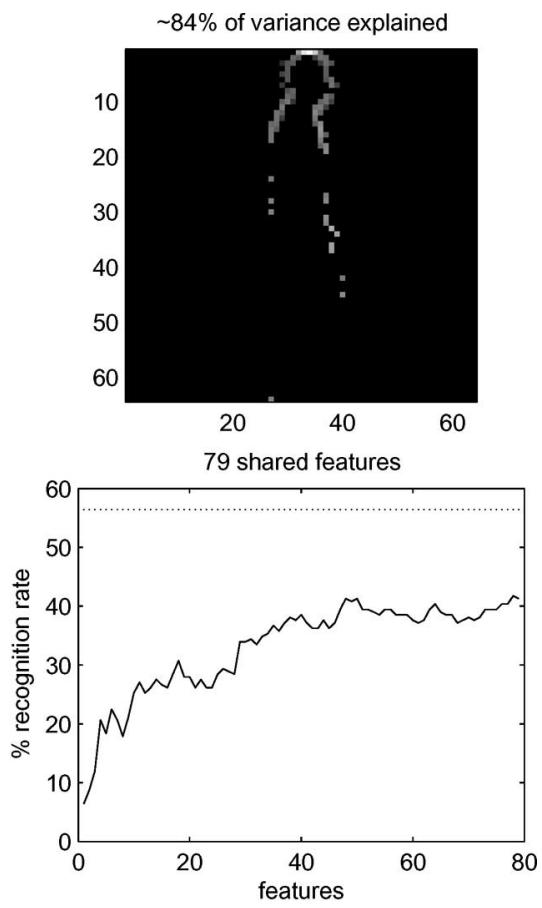

Fig. 7. Analyzing the potency of silhouette measures [40].

means that it is not enough for a differential silhouette to include only the static component of gait, in spite of the fact that static components of gait account for $84 \%$ of explained variance. This reveals an intimate relationship between technique and observation: an averaged silhouette reduces the contribution of the legs and this is revealed in the potency of the measures used. The covariate analysis here has included basic factors and not behavioral factors.

\section{MODEL-BASED APPROACHES}

\section{A. Overview}

The model-based approaches aim to derive the movement of the torso and/or the legs. Unlike the silhouettebased approaches this usually concentrates on dynamics, omitting body shape. The distinction of a structural approach is one which uses static parameters illustrated in Fig. 8(a) whereas a model can be the (relative) motion of the angles $(\gamma, \beta$, and $\phi)$ between the limbs, shown in Fig. 8(b).

BenAbdelkader et al.'s approach using self similarity and the use of structural stride parameters (stride and cadence) [3] is a prime example of a model-based approach which uses structural measures. Cadence was estimated via periodicity; stride length was estimated as the ratio of the distance traveled (given calibration) to the number of steps taken. By analysis on the UMD data, the variation in stride length with cadence was found to be linear and unique for different people, and was used not just for recognition, but also for verification.

Bobick et al. from GaTech used structural human stride parameters [5] which is the other example of a structural model-based approach. The method used the action of walking to derive relative body parameters which described the subject's body and stride. The within-class and between-class variation were analyzed to determine potency and on motion capture data the relative body parameters appeared to have greater discriminatory power

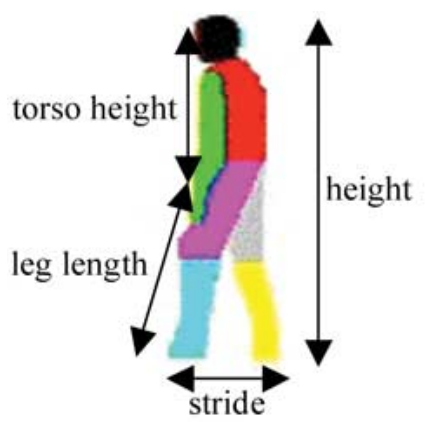

(a)

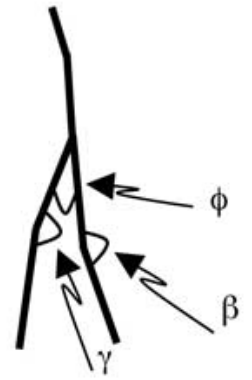

(b)
Fig. 8. Model-based approaches to gait description. (a) Structural. (b) Modeling. 


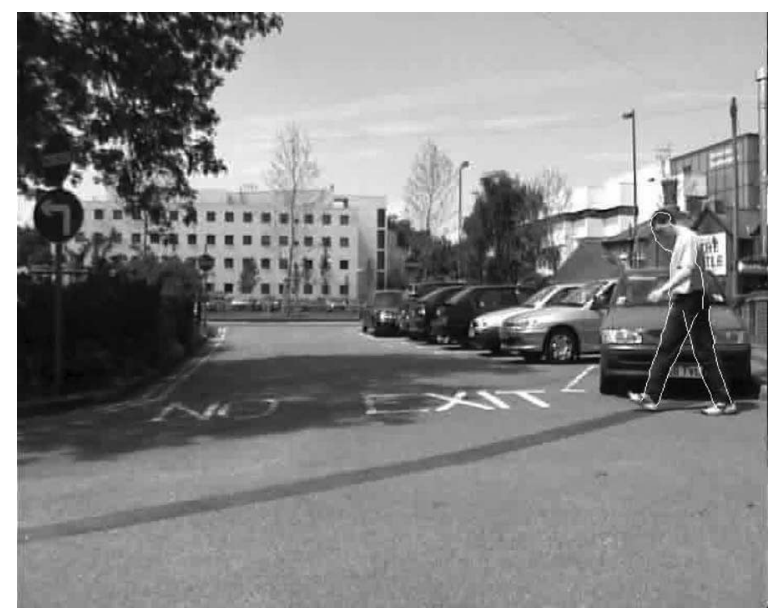

Fig. 9. Extending the model in feature-based analysis.

than the stride parameters. Another structural approach, by Tanawongsuwan et al., used joint angle trajectories, derived by markers placed on joint positions in the legs and on the thorax [36]. A simple method was used to estimate the planar offsets between the marker positions and the underlying skeleton and the variation in joint angles (such as the orientation of the femur relative to the back) with time was then derived. A variance compensated time warping was used to compensate for temporal variations.

Yam et al. extended an earlier model-based system [8] to describe both legs and to handle walking as well as running [46]; an alternative model-based system uses evidence gathering as an initial step, followed by modelbased analysis driven by anatomical constraints and data and evaluated on the Southampton dataset with an analysis of feature potency [41] was developed by Wagg et al.

In Wang's other study [44], a model-based approach derived the dynamic information of gait by using a Condensation framework to track the walker and to recover joint-angle trajectories of lower limbs. The human body model used fourteen rigid body parts, including upper and lower torso, neck, two upper arms, two lower arms, two thighs, two lower legs, two feet, and a head, each of which was represented by a truncated cone except for the head, which was represented by a sphere. Tracking was used to derive joint angle trajectories which were normalized as in an earlier manner [36]. The static body information is derived from temporal pose changes of the segmented moving silhouettes which were represented as an associated sequence of complex vector configurations and were then analyzed using the Procrustes shape analysis method to obtain a compact appearance representation. Both the static and dynamic cues may be used independently for recognition and were also fused on the decision level using different combinations of rules to improve the identification and verification performance. Experimental results confirmed that body angle trajectories are indeed useable for recognition, as expected.

Zhang et al.'s approach [48] concerned the change in orientation of human limbs. The extraction is model-based and the description is structural making this a blend of the two model-based approaches described so far. The lower limbs were represented by trapezoids and the upper body was planar without the arms. Given distances normalized by height of the thorax, the human body posture was represented by a set of distance measurements and inclinations of its constituent parts. The gait features were extracted from gait sequences by the Metropolis-Hastings method to match body parts to the image data. The sequence fit was achieved by minimizing an energy functional which allows for derivation of elevation angles which describe dynamics of gait and trajectories of joint positions which describe spatiotemporal history. The approach thus centered on capturing temporal differences by extracting the elevation of the knee and ankle and the width at the knees and ankles. As these are periodic, they were described by Fourier analysis and then classified via an HMM. The procedure was evaluated on the CMU Mobo and on the NIST databases and shown to have discrimination capability, with better results on the Mobo database. Clearly it enjoys the advantages of model-based techniques in that the data used for classification is intimately linked to gait itself.

Again, there are emergent studies of the potency of the various model-based measures which is important for camera placement in application and for development of new recognition techniques, here for an extended model [41] shown in Fig. 9. The recognition measures were analyzed by using ANOVA and for the performance on the Southampton indoor and outdoor datasets [41]. This gives for an analysis of potency, shown in Table 3, with the

\begin{tabular}{|l|l|l|l|l|}
\hline \multicolumn{4}{|l|}{ Indoor Dataset } & Outdoor Dataset \\
\hline Rank & Feature & F-statistic & Feature & F-statistic \\
\hline 1 & Lower knee width & 239.9 & Lower knee width & 62.1 \\
\hline 2 & Ankle width & 202.2 & Gait frequency & 56.4 \\
\hline 3 & Gait frequency & 168.1 & Ankle width & 47.5 \\
\hline 4 & Upper knee width & 85.7 & Upper knee width & 46.5 \\
\hline 5 & Hip width & 78.1 & Hip width & 35.5 \\
\hline
\end{tabular}


highest F-statistic giving greatest discriminatory capability and hence the highest rank. This is then similar to the earlier analyses of potency of silhouette measures and suggests that the majority of the system's discriminatory capability is derived from gait frequency (cadence) and from some static shape parameters. These shape parameters will be highly dependent on clothing, which may limit the utility of performing recognition solely on the basis of these parameters. These results may in part explain why some approaches using primarily static parameters [5] or cadence [3] achieve good recognition capability from few parameters. There is a significant reduction in discriminatory capability in the outdoor dataset compared to the indoor dataset, resulting from the lower extraction accuracy, but there is still a strong case for recognition potential using this data.

\section{CONCLUSIONS AND FUTURE WORK}

\section{A. Overall Conclusions}

The unique advantage of gait is that it can be used at a distance when other biometrics could be obscured or at too low a resolution to be perceived. Gait can be perceived for identification purposes by analyzing a sequence of images using extant or new computer vision techniques. Automatic recognition by gait is now in a position where its properties and potency are comparable with other biometrics; it is very encouraging that the progression to much larger databases has been accompanied by recognition performance that confirms that people can indeed be recognized by their gait. The studies have concentrated not only on the possibility of recognizing someone by their gait, but also on the uncertainty associated with identification. Two main classes of approach have emerged, as common with many applications of pattern recognition: to either use ensemble statistics or to model features, both can be used to achieve high recognition rates on the available databases. The current databases and techniques arose mainly during DARPA's Human ID at a Distance program; the databases test a range of application capabilities and lessons learned from developments in other biometrics have led to the inclusion of covariate data for evaluation of application potential. The new techniques have been deployed to good effect on the new databases, which contain at least 100 subjects. Further to the study of covariates, researchers have addressed the potency of the measures made of gait to determine which factors contribute most to recognizing people by their body shape and how it moves. Other factors germane to the study of gait include viewpoint invariance and pure 3-D approaches and we anticipate further developments to be made in these. The study of covariates is innate to pattern recognition and we look forward to approaches which are not only invariant to deployment, but also to the covariateswith an especial consideration of time, since this is innate to any behavioral biometric. In application it is not unlikely that these approaches, and other studies in computer vision, will lead to the emergence of markerless systems for analysis of human gait for other purposes, such as for medical diagnosis and therapy. This will alleviate some of the difficulty in deployment of gait analysis and these studies have already started. Naturally, deploying gait as a biometric implies processing sequences of images and could not even have been addressed without the continuing advance in processing power and availability of storage. In this respect we look forward to the opportunities that will arise from the improvements in computational capabilities. In all, it has been fascinating to have seen a biometric evolve from a nascent stage when it appeared possible to recognize people by the way they walk, though only on very small databases, to the current very sophisticated techniques which can handle demanding databases to good effect. As such, we look forward to developments in this emergent and fascinating new biometric.

\section{B. Further Work}

As gait is fundamental to human motion, it is not unlikely that gait could find deployment in many other areas. In surveillance, gait has yet to find use and this is in part due to development of technique. It is only recently that gait has been demonstrated to be able to recognize people on large databases of outdoor data. Even then, its use in surveillance video analysis for forensic purpose mandates the ability to perform 3-D analysis from images derived by a single camera. There are viewpoint invariant approaches and the model-based approaches do have limited viewpoint invariance. These are insufficiently generalized for forensic analysis. There is a consideration that the likelihood of error, in analyzing single frames derived from low-resolution surveillance video, is likely to be sufficiently low so as to preclude forensic use. This error will be reduced by analyzing sequences of video data, though experimentation will be required to determine by how much this occurs. As such, we await development for forensic deployment, though early and very recent studies have shown that "Surveillance images from a bank robbery were analyzed and compared with images of a suspect. Based on general bodily features, gait, and anthropometric measurements, we were able to conclude that one of the perpetrators showed strong resemblance to the suspect." [26]. There is of course concern at such developments: DARPA's HumanID at a Distance program was even nominated as "Privacy Villain of the Week" in 2002.

It is much more likely that the use of gait in surveillance video will be to signal events likely to be of interest. By way of example, the ability to discriminate between litter blowing into a perimeter fence and a person walking near it or climbing it would make an operator focus on relevant data, and so long as the false alarm rate is 
sufficiently low then this will improve overall response of a surveillance system. In this respect some of the approaches to gait are already sufficiently simple, such as the averaged silhouette or the use of area, to make video-rate analysis possible. Certainly, the frameworks require viewpoint invariance and ability to disambiguate articulated motion but this is a much simpler target than full recognition by gait. These technologies are largely ready for such deployment, now.
Use in animation is likely to be longer away. Computer vision researchers have been synthesizing human faces for some time, and this has been used for face recognition (by using it as a vector to achieve viewpoint invariance). Further, approaches have moved to realistic depiction of the human shape and these could use gait biometrics to better model motion, reflecting the wider contribution possible from these studies and the basic nature of gait in human movement.

\section{REFERENCES}

[1] C. BenAbdelkader, R. Cutler, and L. Davis, "Eigengait: Motion-based recognition of people using image self-similarity," in Proc. 3rd Int. Conf. Audio- and Video-Based Biometric Person Authentication, 2001, vol. 2091, Lecture Notes in Computer Science, pp. 284-294.

[2] _ - "Motion-based recognition of people in EigenGait space," in Proc. IEEE Face and Gesture Recognition, 2002, pp. 254-259.

[3] _ - "Stride and cadence as a biometric in automatic person identification and verification," in Proc. IEEE Face and Gesture Recognition, 2002, pp. 372-377.

[4] B. Bhanu and J. Han, "Human recognition on combining kinematic and stationary features," in Proc. Int. Conf. Audio- and Video-Based Biometric Person Authentication, 2003, vol. 2688, Lecture Notes in Computer Science, pp. 600-608.

[5] A. F. Bobick and A. Y. Johnson, "Gait recognition using static, activity-specific parameters," in Proc. IEEE Computer Vision and Pattern Recognition 2001, vol. 1, pp. 423-430.

[6] J. E. Boyd, "Synchronization of oscillations for machine perception of gaits," Comput. Vis. Image Understand., vol. 96, pp. 35-59, 2004.

[7] R. Collins, R. Gross, and J. Shi, "Silhouette-based human identification from body shape and gait," in Proc. IEEE Int. Conf. Face and Gesture Recognition '02, pp. 366-371.

[8] D. Cunado, M. S. Nixon, and J. N. Carter, "Automatic extraction and description of human gait models for recognition purposes," Comput. Vis. Image Understand., vol. 90, no. 1, pp. 1-41, 2003.

[9] J. E. Cutting and L. T. Kozlowski, "Recognizing friends by their walk," Bull. Psychonom. Soc., vol. 9, no. 5, pp. 353-356, 1977

[10] J. P. Foster, M. S. Nixon, and A. Prugel-Bennet, "Automatic gait recognition using area-based metrics," Pattern Recognit. Lett., vol. 24, pp. 2489-2497, 2003.

[11] M. G. Grant, J. D. Shutler, and M. S. Nixon, "Analysis of a human extraction system for deploying gait biometrics," in IEEE Southwest Symp. Image Analysis and Interpretation (SSIAI '04), pp. 46-50.

[12] R. Gross and J. Shi, The CMU Motion of Body (MoBo) Database, Robotics Institute, Carnegie Mellon Univ., Pittsburgh, PA, Tech. Rep. CMU-RI-TR-01-18, Jun. 2001. [Online]. Available: http://www.ri.cmu.edu/pubs/ pub_3904.html

[13] J. Han and B. Bhanu, "Statistical feature fusion for gait-based human recognition," in Proc. IEEE Computer Vision and Pattern Recognition 2004, vol. 2, pp. II-842-II-847.

[14] J. B. Hayfron-Acquah, "Automatic gait recognition by symmetry analysis," Ph.D. dissertation, Univ. Southampton, Southampton, U.K., 2003.

[15] J. B. Hayfron-Acquah, M. S. Nixon, and J. N. Carter, "Automatic gait recognition by symmetry analysis," Pattern Recognit. Lett., vol. 24, no. 13, pp. 2175-2183, 2003.

[16] P. S. Huang, C. J. Harris, and M. S. Nixon, "Recognizing humans by gait via parametric canonical space," Artif. Intell. Eng., vol. 13, no. 4, pp. 359-366, 1999.

[17] G. Johannson, "Visual perception of biological motion and a model for its analysis," Perception Psychophys., vol. 14, pp. 201-211, 1973.

[18] A. Kale, A. N. Rajagopalan, A. Sundaresan, N. Cuntoor, A. Roy-Chowdhury, V. Kruger, and R. Chellappa, "Identification of humans using gait," IEEE Trans. Image Process., vol. 13, no. 9, pp. 1163-1173, Sep. 2004.

[19] C.-S. Lee and A. Elgammal, "Gait style and gait content: Bilinear models for gait recognition using gait re-sampling," in Proc. IEEE Int. Conf. Face and Gesture Recognition '04, pp. 147-152.

[20] L. Lee and W. E. L. Grimson, "Gait analysis for recognition and classification," in Proc. IEEE Int. Conf. Face and Gesture Recognition '02, pp. 155-162.

[21] J. Little and J. Boyd, "Recognizing people by their gait: The shape of motion," Videre, vol. 1, no. 2, pp. 1-32, 1998.

[22] Y. Liu, R. Collins, and Y. Tsin, "Gait sequence analysis using frieze patterns," in Proc. Eur. Conf. Computer Vision, 2002, pp. 657-671.

[23] Z. Liu, L. Malave, A. Osuntugun, P. Sudhakar, and S. Sarkar, "Towards understanding the limits of gait recognition," in Proc. SPIE Int. Symp. Defense and Security: Biometric Technology for Human Identification, 2004, pp. 195-205.

[24] Z. Liu and S. Sarkar, "Simplest representation yet for gait recognition: Averaged silhouette," in Proc. 17th Int. Conf. Pattern Recognition, 2004, vol. 4, pp. 211-214.

[25] H. Murase and R. Sakai, "Moving object recognition in eigenspace representation: Gait analysis and lip reading," Pattern Recognit. Lett., vol. 17, pp. 155-162, 1996.

[26] N. Lynnerup and J. Vedel, "Person identification by gait analysis and photogrammetry," J. Forensic Sci., vol. 50, no. 1, pp. 112-118, Jan. 2005.

[27] M. P. Murray, "Gait as a total pattern of movement," Amer. J. Phys. Med., vol. 46, no. 1, pp. 290-332, 1967.

[28] M. P. Murray, A. B. Drought, and R. C. Kory, "Walking patterns of normal men," J. Bone Joint Surg., vol. 46-A, no. 2, pp. 335-360, 1964.

[29] M. S. Nixon, T. N. Tan, and R. Chellappa, Human Identification Based on GaitNew York: Springer, 2005.

[30] S. A. Niyogi and E. H. Adelson, "Analyzing and recognizing walking figures in XYT," in
Proc. IEEE Computer Vision and Pattern Recognition, 1994, pp. 469-474.

[31] S. Sarkar, P. J. Phillips, Z. Liu, I. R. Vega, P. Grother, and K. Bowyer, "The humanID gait challenge problem: Data sets, performance and analysis," IEEE Trans. Pattern Anal. Mach. Intell., vol. 27, no. 2, pp. 162-177, Feb. 2005.

[32] J. D. Shutler and M. S. Nixon, “Zernike velocity moments for description and recognition of moving shapes," in Proc. BMVC 2001, pp. 705-714.

[33] J. D. Shutler, M. G. Grant, M. S. Nixon, and J. N. Carter, "On a large sequence-based human gait database, special session on biometrics," in Proc. 4th Int. Conf. Recent Advances in Soft Computing, 2002, pp. 66-72.

[34] S. V. Stevenage, M. S. Nixon, and K. Vince, "Visual analysis of gait as a cue to identity," Appl. Cogn. Psychol., vol. 13, no. 6, pp. 513-526, 1999.

[35] A. Sundaresan, A. Roy-Chowdhury, and R. Chellappa, "A hidden Markov model based framework for recognition of humans from gait sequences," in Proc. IEEE Int. Conf. Image Processing, 2003, pp. 143-150.

[36] R. Tanawongsuwan and A. Bobick, "Gait recognition from time-normalized joint-angle trajectories in the walking plane," in Proc. IEEE Computer Vision and Pattern Recognition 2001, pp. II-726-II-731.

[37] D. Tolliver and R. Collins, "Gait shape estimation for identification," in Proc. Int. Conf. Audio and Video-Based Biometric Person Authentication, 2003, vol. 2688, Lecture Notes in Computer Science, pp. 734-742.

[38] A. Veeraraghavan, A. Roy-Chowdhury, and R. Chellappa, "Role of shape and kinematics in human movement analysis," in Proc. IEEE Computer Vision and Pattern Recognition 2004, vol. 1, pp. I-730-I-737.

[39] I. R. Vega and S. Sarkar, "Statistical motion model based on the change of feature relationships: Human gait-based recognition," IEEE Trans. Pattern Anal. Mach. Intell., vol. 25, no. 10 , pp. 1323-1328, Oct. 2003.

[40] G. Veres, J. N. Carter, and M. S. Nixon, "What image information is important in silhouette-based gait recognition," in Proc. IEEE Computer Vision and Pattern Recognition 2004, vol. 2, pp. 776-782.

[41] D. K. Wagg and M. S. Nixon, "On automated model-based extraction and analysis of gait," in Proc. IEEE Int. Conf. Face and Gesture Recognition '04, pp. 11-16.

[42] L. Wang, T. N. Tan, W. M. Hu, and H. Z. Ning, "Automatic gait recognition based on statistical shape analysis," IEEE Trans. Image Proc., vol. 12, no. 9, pp. 1120-1131, Sep. 2003.

[43] L. Wang, T. Tan, H. Z. Ning, and W. M. Hu, "Silhouette analysis-based gait recognition for human identification," IEEE Trans. Pattern 
Anal. Mach. Intell., vol. 25, no. 12, pp. 1505-2528, Dec. 2003.

[44] L. Wang, T. Tan, H. Ning, and W. Hu, "Fusion of static and dynamic body biometrics for gait recognition," IEEE Trans. Circuits Syst. Video Technol., vol. 14, no. 2, pp. 149-158, Feb. 2004.

[45] D. Winter, The Biomechanics and Motor Control of Human Gait, 2nd ed. Ottawa, ON, Canada: Waterloo Biomechanics, 1991.
[46] C.-Y. Yam, M. S. Nixon, and J. N. Carter, "Automated person recognition by walking and running via model-based approaches," Pattern Recognit., vol. 37, no. 5, pp. 1057-1072, 2004.

[47] J. Zhang, R. Collins, and Y. Liu, "Representation and matching of articulated shapes," in Proc. IEEE Computer Vision and Pattern Recognition 2004, Washington, 2004.
[48] R. Zhang, C. Vogler, and D. Metaxas, "Human gait recognition," in Proc. IEEE Computer Vision and Pattern Recognition 2004, vol. 2, pp. 342-349.

[49] G. Zhao, R. Chen, G. Liu, and L. Hua, "Amplitude spectrum-based gait recognition," in Proc. IEEE Int. Conf. Face and Gesture Recognition '04, pp. 23-28.

\section{ABOUT THE AUTHORS}

Mark S. Nixon (Member, IEEE) is the Professor of Computer Vision, University of Southampton, Southampton, U.K. His team develops new techniques for static and moving shape extraction which have found application in automatic face and automatic gait recognition and in medical image analysis. His team were early workers in face recognition, later came to pioneer gait recognition and more recently joined the pioneers of ear biometrics. His vision book, co-

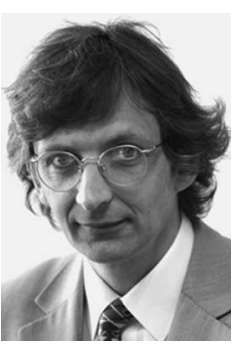
written with Alberto Aguado, Feature Extraction and Image Processing was published in 2002 by Butterworth and with Tieniu Tan and Rama Chellappa, his new book Human ID Based on Gait which is part of the new Springer Series on Biometrics, was published in 2005. His research interests are in image processing and computer vision.

Dr. Nixon chaired BMVC 98, and with Josef Kittler he chaired the Audio Visual Biometric Person Authentication (AVBPA 2003), was Publications Chair for the International Conference on Pattern Recognition (ICPR 2004) and the IEEE 7th International Conference on Face and Gesture Recognition FG2006. Amongst research contracts, he was Principal Investigator with John Carter on the DARPA-supported project Automatic Gait Recognition for Human ID at a Distance.
John N. Carter (Member, IEEE) received the B.A. degree in experimental physics from Trinity College, Dublin, Ireland, and the Ph.D. degree in astrophysics from the University of Southampton, Southampton, U.K.

In 1985, he changed disciplines and joined the Department of Electronic Engineering, also at Southampton, as a Lecturer researching in signal and image processing. He is currently a Senior Lecturer in the Information: Signals, Images and

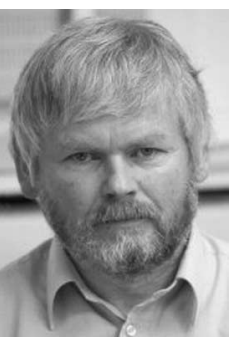
Systems Research Group. He has worked on programs as diverse as diesel engine diagnostics and vocal tract imaging. A recent success in this field has been the development of a new dynamic form of magnetic resonance imaging, which makes it possible to reconstruct high time resolution multiplanar views of the human vocal tract while a subject is repeating a short phrase. His current major interests are in the general area of four-dimensional image processing - that is, analyzing sequences of images to extract both two- and three-dimensional features, exploiting coherence over the whole sequence, i.e., imposing simple smoothness and continuity constraints. This has applications in object tracking and feature detection, where it is tolerant of high levels of noise and missing data. This has found application in biometrics, in particular, automatic gait analysis. 